\title{
Vacunación antigripal en la farmacia comunitaria: opinión de pacientes y farmacéuticos
}

\author{
N. Floro Andrés-Rodríguez ${ }^{1,3}$, Rocío Mera-Gallego ${ }^{1,3}$, Álex Piñeiro-Abad ${ }^{1,3}$, Adrián Acuña-Ferradanes ${ }^{1,3}$, \\ Inés Mera-Gallego ${ }^{1,2}$, Patricia García-Rodríguez ${ }^{1,3}$, J. Carlos Andrés-Iglesias ${ }^{1,3}$, José A. Fornos-Pérez ${ }^{1,3}$ \\ 1. Farmacéuticos comunitarios en la provincia de Pontevedra. 2. Farmacéutica comunitaria en Maella. Zaragoza. 3. Grupo Berbés de Investigación y Docencia.
}

\section{PALABRAS CLAVE}

Vacuna antigripal, farmacéuticos comunitarios, pacientes, actitud del personal de salud, rechazo a vacunarse, servicio profesional farmacéutico

\section{ABREVIATURAS}

CAP: centro de atención primaria CDCP: Centers for Diseases Control and Prevention

COF: Colegio de Farmacéuticos COFPO: Colegio Oficial de Farmacéuticos de Pontevedra FC: farmacéuticos comunitarios OCDE: Organización para la Cooperación y el Desarrollo Europeo OMS: Organización Mundial de la Salud

PRC: patologías que suponen un riesgo de sufrir complicaciones $\mathrm{R}$ : rural

SEFAC: Sociedad Española de Farmacia Familiar y Comunitaria SERGAS: Servizo Galego de Saúde SU: semiurbano

$\mathrm{U}$ : urbano

\section{KEYWORDS}

Influenza vaccine, community pharmacists, patients, attitude of health personnel, vaccination refusal, pharmaceutical services

\section{RESUMEN}

Objetivos: Conocer la opinión de pacientes y farmacéuticos comunitarios (FC) sobre la posible administración de la vacuna antigripal en farmacias y comprobar si una campaña educativa consigue mejorar la intención de vacunación.

Material y métodos: Estudio observacional con intervención educativa.

Sujetos: usuarios de la farmacia mayores de 45 años. FC ejercientes en la provincia de Pontevedra.

Variables pacientes: Demográficas, pertenencia a grupo de riesgo, intención de vacunación antes/después de la intervención, opinión sobre la vacunación en la farmacia.

Variables FC: Demográficas, opinión sobre la vacunación por el FC, necesidad de formación, a quién correspondería la acreditación.

Procedimiento: Al participante se le explicaba el propósito del estudio y a los no vacunados se les informaba de los beneficios de vacunarse. A 200 FC de Pontevedra se les envió un cuestionario ad hoc.

Resultados: Se entrevistó a 157 usuarios, $134(85,4 \%)$ a favor de la vacunación por el FC. Sin diferencias entre sexos, estudios, ni pertenencia a grupo de riesgo. Tras la intervención, de 92 que no pensaban vacunarse, 27 (29,6\%) decidieron acudir a hacerlo.

93 respuestas de FC (46,5\%). 63 (67,7\%) a favor de la vacunación por el FC, con diferencias significativas entre titulares/adjuntos $(p<0,05)$ y socios/no socios de SEFAC $(p<0,05) .82$ $(88,2 \%)$ FC opinan que se necesita formación específica para la actividad.

Conclusiones: La opinión de que la farmacia debe ser punto de administración de la vacuna antigripal es mayoritaria entre pacientes y farmacéuticos, significativamente mayor en titulares y socios de SEFAC. La intención de vacunación mejoró en un 29,4\%.

\section{Influenza vaccination in the community pharmacy: opinion of patients} and pharmacists

\section{ABSTRACT}

Objectives: To find out the opinion of patients and community pharmacists (CPs) regarding the possible administration of the influenza vaccine in pharmacies and to verify whether an educational campaign manages to improve vaccination intention.

Material and methods: Observational study with educational intervention.

Subjects: pharmacy users over 45 years old. Practicing CPs in the province of Pontevedra.

Patient variables: demographic, membership of the risk group, vaccination intention before/after the intervention, opinion about being vaccinated at the pharmacy.

$\mathrm{CP}$ variables: demographic, opinion on vaccination by the $\mathrm{CP}$, need for training, to whom the accreditation would correspond.

Procedure: the participant was explained the purpose of the study and those who had not been vaccinated were informed of the benefits of getting vaccinated. Two hundred CPs from Pontevedra were sent an ad hoc questionnaire.

Results: One hundred fifty-seven users were interviewed, 134 (85.4\%) were in favor of being vaccinated by the $\mathrm{CP}$. No differences between sexes, studies, or belonging to a risk group. After the intervention, of 92 who did not plan to get vaccinated, 27 (29.6\%) decided to go and do it.

93 responses from CPs (46.5\%). 63 (67.7\%) in favor of the vaccination by CP, with significant differences between owners/assistants $(\mathrm{p}<0.05)$ and members/non-members of SEFAC $(\mathrm{p}<0.05) .82(88.2 \%)$ CPs think that specific training is needed for the activity.

Conclusions: The majority of patients and pharmacists believe that the pharmacy should be the point of administration of the influenza vaccine. This is significantly higher among owners and members of SEFAC. Vaccination intention improved by $29.4 \%$.

Financiación: ninguna.

Conflicto de intereses: ninguno.

Cite este artículo como: Andrés-Rodriguez NF, Mera-Gallego R, Piñeiro-Abad A, Acuña-Ferradanes A, Mera-Gallego I, Garcia-Rodriguez P, Andrés-Iglesias JC, Fornos-Pérez JA. Vacunación antigripal en la farmacia comunitaria: opinión de pacientes y farmacéuticos. Farmacéuticos Comunitarios. 2018 Sep 28; 10(3):15-24. doi:10.5672/FC.2173-9218.(2018/ Vol 10).003.03 


\section{Introducción}

La personas afectadas por gripe (Influenza) sufren un cuadro de aparición brusca con fiebre (entre $38 \mathrm{y}$ $40^{\circ} \mathrm{C}$ ), de unos 3 a 7 días de duración, acompañada de cefalea, congestión nasal, tos generalmente seca, falta de apetito, dolor al deglutir, cansancio, dolor muscular y articular y malestar general [1-3]. La mayoría se recuperan en 1 o 2 semanas, aunque en algunos casos pueden presentarse complicaciones, generalmente patologías respiratorias entre las que destacan bronquitis y neumonía, producida por infecciones secundarias pulmonares, más frecuente en mayores, pacientes con cardiopatías previas, enfermedades pulmonares crónicas, diabéticos o inmunodeprimidos [1-3].

El gran impacto social de la gripe la convierte en un auténtico problema de salud pública. Las epidemias anuales causan de 3 a 5 millones de casos de enfermedad grave en el mundo y unos 250.000 a 500.000 fallecimientos [4]. En España, se infectan de gripe cada año entre $3 \mathrm{y}$ 3,5 millones de individuos, lo cual significa unas 7.800-8.000 personas por cada 100.000 habitantes [5]. En la temporada 2016-17 se notificaron 2.874 casos graves hospitalizados confirmados de gripe en 18 comunidades autónomas, de los que 619 (22\%) fueron ingresados en UCI y 464 (17\%) fallecieron. La mediana de edad fue de 76 años con un 74\% mayores de 64 años [6].

La vacunación antigripal es la medida preventiva más eficaz, especialmente entre la población con patologías que suponen un riesgo de sufrir complicaciones (PRC). Es gratuita en los centros de salud dentro del período de campaña de cada año, aunque también se puede adquirir en las farmacias previa prescripción médica. Está recomendada a los mayores de 65 años, las personas con alto riesgo de sufrir complicaciones en caso de padecer la gripe: enfermedad crónica cardiovascular, pulmonar, renal, hepática o neuromuscular, diabéticos, inmunodeprimidos, embarazadas. También las personas en contacto con estos grupos de alto riesgo, ya que pueden transmitírsela: trabajadores de centros sanitarios 0 geriátricos, policías, bomberos, protección civil [7]. Una vez administrada la vacuna, la protección eficaz comienza después de aproximadamente dos se- manas y dura hasta 6 meses, lo que hace necesario repetir anualmente la vacunación [8]. Aunque la efectividad de la vacunación parece ser muy variable (23\% [9], 50-60\% [10], 30-80\% [11]) en función de la edad, el grado de inmunocompetencia y la coincidencia de los componentes de la vacuna con los virus de la temporada, todas las organizaciones e instituciones nacionales y supranacionales recomiendan su empleo.

El Consejo Interterritorial del Sistema Nacional de Salud en nuestro país se planteó como objetivo 201718 alcanzar el 65\% en el grupo de edad mayor o igual de 65 años [12] con el fin de acercarse al objetivo establecido por la Organización Mundial de la Salud y por la Comisión Europea de lograr coberturas de, al menos, el 75\% en este grupo etario y superar una cobertura del 40\% en el grupo de profesionales sanitarios [12].

Lamentablemente los objetivos de la OMS no se alcanzan en España ni en ningún país de nuestro entorno socio-económico. En Estados Unidos los Centros para la Prevención y Control de Enfermedades (CDCP) cifran la cobertura vacunal en adultos en el $43,3 \%$ en $2016 / 17$, con un 65,3\% en los mayores de 65 años, habiendo aumentado desde la anterior campaña en el 1,9\% [13]. En la campaña actual y a fecha de 28/12/2017, los datos del US National Vaccine Program Office contabilizan una tasa de vacunación del 42,5\% de la población general con un 45,6\% en los mayores de 65 años y un 26,4\% en los menores de 65 años [14].

Según datos de la Organización para la Cooperación y el Desarrollo Europeo (OCDE) [15], en 2015 solo dos países europeos superaron el 65\%: Gran Bretaña $(71,1 \%)$ y Holanda $(66,8 \%)$.

En España se pasó del 56,1\% en 2015/16 a 55,6 en 2016/17 [15], siendo entre los profesionales sanitarios de solo el 31,5\% [15]. En la última campaña (2017/18), con datos correspondientes al 20/12/2017, se ha vacunado en nuestro país el 23,5\% del tramo entre 18-65 años, el 55,3\% de los mayores de 65 años y el 38,1\% de los profesionales sanitarios [16].

El farmacéutico comunitario, además de vacunarse, al tratarse de un profesional sanitario en contacto con pacientes de riesgo a los que puede transmitirla, participa en campañas de difusión y promoción de la vacunación antigripal entre los usuarios de su farmacia. Sociedades científicas y algunos Colegios de Farmacéuticos (COF) han ofrecido a las autoridades sanitarias competentes la colaboración en las campañas anuales mediante la administración de las vacunas por los farmacéuticos comunitarios en las farmacias, como se hace con notable éxito en otros países. En abril del año 2015 el COF de Pontevedra (COFPO) propuso a la Consellería de Sanidade/Servizo Galego de Saúde (SERGAS) un programa que consistía en la participación de las farmacias comunitarias de la provincia en la campaña de vacunación antigripal mediante su inclusión como un punto de administración de la vacuna. Se pretendía contribuir a mejorar la baja tasa de vacunación antigripal en la provincia de Pontevedra, por debajo del 50\% en la población mayor de 60 años, concienciando sobre los beneficios de la vacunación y administrando la vacuna a los ciudadanos. La frontal oposición de médicos y enfermeros apoyada en argumentos partidarios, ideológicos y de defensas sectoriales, ignorando experiencias muy positivas para la salud de la población, impidió el éxito de la propuesta.

Otros países (Canadá, Estados Unidos, Australia, Reino Unido, Irlanda, Suiza, Portugal, Dinamarca, etc.) han seguido las recomendaciones de expertos e instituciones sanitarias en el sentido de ampliar el acceso a la vacunación antigripal en ámbitos no tradicionales en especial las farmacias comunitarias [17-23].

En el presente trabajo pretendemos conocer la opinión de los pacientes y de los farmacéuticos comunitarios sobre la posible implantación de un servicio de vacunación antigripal en las farmacias comunitarias por FC acreditados, además de evaluar la efectividad de una campaña educativa en la mejora de la intención de vacunación.

\section{Objetivos}

- Conocer la opinión de la población sobre la posible administración de las vacunas de la gripe en las farmacias comunitarias por farmacéuticos acreditados.

- Comprobar si la intervención del farmacéutico en una campaña de educación para la salud consigue 
mejorar la intención de vacunación de la población.

- Conocer la opinión de los farmacéuticos comunitarios sobre la posible administración de las vacunas de la gripe en las farmacias comunitarias.

- Saber si hay diferencias en la opinión entre edad, sexo, localidades, categorías laborales y pertenencia o no a la Sociedad Española de Farmacia Familiar y Comunitaria (SEFAC).

- Conocer quién debería, en opinión de pacientes y farmacéuticos, abonar la prestación del servicio.

\section{Material y métodos Diseño}

Estudio observacional transversal con intervención educativa realizado en cuatro farmacias comunitarias de la provincia de Pontevedra durante el mes de noviembre de 2017 (pacientes) y en marzo-abril de 2018 (farmacéuticos).

\section{Sujetos}

Usuarios de la farmacia mayores de 45 años que accedieron a participar en el estudio y cuya situación psicopatológica o social les permitiese la comprensión de las preguntas del farmacéutico investigador. FC que ejercen en la provincia de Pontevedra y accedieron a participar en el estudio.

\section{Cálculo del tamaño muestral}

Encuesta a pacientes: para conseguir una precisión del 10,0\% en la estimación de una proporción mediante un intervalo de confianza asintótico normal al 95\% bilateral, asumiendo que la proporción es del $50 \%$ en la encuesta a pacientes, resultó necesario incluir 96 unidades experimentales en el estudio. En la encuesta a farmacéuticos, para las mismas condiciones, una en población finita de 1.191 FC ejercientes en Pontevedra y una proporción esperada del 70\%, fue necesario incluir 76 unidades experimentales en el estudio.

\section{Procedimiento}

Se ofreció participar en el estudio al primer paciente de la mañana y de la tarde que cumplían los criterios de inclusión hasta completar el período de estudio. La captación de los sujetos se realizó en el mostrador de la farmacia, donde se explicaba al participante el propósito de la campaña de promoción de la vacunación antigripal y a los usuarios que todavía no se habían vacunado se les informaba de los beneficios de la vacunación mediante un argumentario específico diseñado por el personal del Departamento Profesional del COFPO, y se cumplimentaba el cuestionario de recogida de datos. La entrevista se realizó en el momento de la dispensación, sin recoger datos identificativos de los pacientes.

Para la encuesta a farmacéuticos se diseñó un cuestionario ad hoc, que se envió por correo electrónico a 200 FC de Pontevedra. Se realizó una aleatorización estratificada en función de la distribución de los FC ejercientes en municipios rurales (R), semiurbanos (SU) y urbanos (U), elegidos al azar en un listado de municipios de la provincia .

En el anexo 1 se muestran las encuestas cumplimentadas por pacientes y farmacéuticos.

\section{Variables}

\section{Encuesta a pacientes}

- Variables demográficas: sexo (hombre/mujer), nivel de estudios (sin estudios/primarios/secundarios-FP/universitarios) y tipo de riesgo $(<60$ años $\sin \mathrm{PRC} /<60$ años con $\mathrm{PRC} />60$ años sin $\mathrm{PRC} />60$ años con PRC/sujeto en contacto con personas con PRC/mujer embarazada).

- Situación con respecto a la administración de la vacuna antigripal de este año (vacunado/no vacunado). Tiene pensado hacerlo (sí/no).

- Intención de acudir al centro de atención primaria (CAP) para recibir la vacuna tras la información recibida (sí/no/duda).

- Opinión sobre la posibilidad de administración de la vacuna en la farmacia por el farmacéutico comunitario (sí/no). Motivos (proximidad/accesibilidad/ambos/otro).

- Opinión sobre quién debe abonar el servicio (SERGAS/parte SERGAS parte paciente/SERGAS en grupos de riesgo y paciente en grupos no de riesgo). Importe a pagar $(€)$.
Encuesta a farmacéuticos

- Variables demográficas: sexo (hombre/ mujer), edad (años), categoría de ejercicio profesional (titular/ adjunto), carácter R, SU o U de la localidad de ejercicio, pertenencia o no a SEFAC.

- Opinión sobre la vacunación por el FC (sí/no).

- La necesidad o no de formación (sí/ no).

- A quién correspondería la acreditación (Colegios de Farmacéuticos, SERGAS, SEFAC, otros).

- Quién debería responsabilizarse del abono del servicio (SERGAS/parte SERGAS parte paciente/SERGAS en grupos de riesgo y paciente en grupos no de riesgo).

Expresión de los resultados y análisis estadístico

Se utilizó el programa estadístico SPSS ${ }^{\circledR} 22.0$ para Windows ${ }^{\circledR}$. Los datos cualitativos se expresan como porcentajes y los cuantitativos como media \pm desviación estándar. Los límites de confianza se calculan al 95\%, utilizando los test de chi-cuadrado para el análisis de variables cualitativas, t de Student para variables cuantitativas con distribución normal y Mann-Whitney para variables cuantitativas con distribución no normal. La significación estadística se fijó en $\mathrm{p}<0,05$.

\section{Resultados}

\section{Opinión de los pacientes}

En las cuatro farmacias se realizaron 157 encuestas, sin que se produjese ningún rechazo a participar en el estudio. Las características de la muestra se presentan en la tabla 1 .

$134(85,4 \%)$ de los encuestados creen que la farmacia comunitaria debe ser un punto de administración de la vacuna de la gripe estacional; los otros 23 (14,6\%) prefieren el CAP.

La distribución en función de estar ya vacunados o todavía no se muestra en la tabla 2.

No hubo diferencias significativas en cuanto a la opinión favorable a la vacunación en la FC entre sexos $(p=0,1014)$, estudios $(p=0,6669)$ o características de riesgo $(\mathrm{p}=0,7116)$.

Los motivos por los que los usuarios creen que la farmacia debe ser un punto de administración de la vacuna de la gripe y por los que opinan que no se muestran en la tabla 3. 
Tabla 1 Caracteristicas de la muestra de usuarios encuestados

\begin{tabular}{|c|c|c|c|}
\hline Característica & $\begin{array}{c}\text { Hombres } \\
\text { n (\%) }\end{array}$ & $\begin{array}{c}\text { Mujeres } \\
\text { n (\%) }\end{array}$ & $\begin{array}{l}\text { Total } \\
\text { n (\%) }\end{array}$ \\
\hline Sexo & $58(36,9)$ & $99(63,1)$ & $157(100,0)$ \\
\hline \multicolumn{4}{|l|}{ Nivel de estudios } \\
\hline Sin estudios & $5(8,6)$ & $19(19,2)$ & $24(15,3)$ \\
\hline Primarios & $20(34,5)$ & $41(41,4)$ & $61(38,9)$ \\
\hline Secundarios/FP & $14(24,1)$ & $19(19,2)$ & $33(21,0)$ \\
\hline Universitarios & $19(32,8)$ & $20(20,2)$ & $39(24,8)$ \\
\hline \multicolumn{4}{|l|}{ Riesgo } \\
\hline$<60$ años sin factores de riesgo & $5(8,6)$ & $14(14,1)$ & $19(12,1)$ \\
\hline$<60$ años con factores de riesgo & $14(24,1)$ & $19(19,2)$ & $33(21,0)$ \\
\hline$>60$ años sin factores de riesgo & $14(24,1)$ & $12(12,1)$ & $26(16,6)$ \\
\hline$>60$ años con factores de riesgo & $23(39,7)$ & $46(46,5)$ & $69(43,9)$ \\
\hline $\begin{array}{l}\text { Cuidador o persona con riesgo de } \\
\text { transmisión a personas susceptibles de } \\
\text { complicaciones }\end{array}$ & $2(3,5)$ & $5(5,1)$ & $7(4,5)$ \\
\hline Mujer embarazada & & $3(3,0)$ & $3(1,9)$ \\
\hline
\end{tabular}

Tabla 2 Opinión de los usuarios encuestados sobre la administración en FC en función de estar ya vacunados o todavía no

\begin{tabular}{|l|c|c|c|}
\hline & $\begin{array}{c}\text { Vacunados } \\
\mathrm{n}=46\end{array}$ & $\begin{array}{c}\text { No vacunados } \\
\mathrm{n}=111\end{array}$ & p-valor \\
\hline Administración en la farmacia & $40(87,0 \%)$ & $94(84,7 \%)$ & \multirow{2}{*}{0,7141} \\
\hline Administración en el CAP & $6(13,0 \%)$ & $17(15,3 \%)$ & \\
\hline
\end{tabular}

Tabla 3 Motivos por los que los usuarios creen o no que la farmacia debe ser un punto de vacunación

\begin{tabular}{|c|c|}
\hline \multicolumn{2}{|c|}{$\begin{array}{l}\text { La farmacia debe ser un punto de } \\
\text { vacunación }(n=134)\end{array}$} \\
\hline $\begin{array}{l}\text { Por proximidad y } \\
\text { accesibilidad }\end{array}$ & $85(63,4 \%)$ \\
\hline Porque está más cerca & $27(20,2 \%)$ \\
\hline Por no tener que esperar & $22(16,4 \%)$ \\
\hline \multicolumn{2}{|c|}{$\begin{array}{l}\text { La vacunación debe realizarse en el CAP } \\
(n=23)\end{array}$} \\
\hline Debe vacunar el SERGAS & $11(47,8 \%)$ \\
\hline Le da igual & $2(8,7 \%)$ \\
\hline $\begin{array}{l}\text { "De paso que le hacen el } \\
\text { INR ya se vacuna" }\end{array}$ & $1(4,3 \%)$ \\
\hline $\begin{array}{l}\text { No refieren un motivo } \\
\text { concreto }\end{array}$ & $8(34,8 \%)$ \\
\hline
\end{tabular}

De los 157 encuestados, 111 (70,7\%) no habían sido todavía vacunados de la gripe. El número de vacunados fue mayor entre los de más alto nivel de estudios $(\mathrm{p}=0,0183)$ y entre los que estaban en GR ( $p=0,0141)$. De los 111 no vacunados no pensaban vacunarse 92 (82,9\%), el 58,6\% del total de encuestados, sin diferencias significativas en relación con el nivel de estudios ( $p=0,3182)$ y estar o no en GR ( $p=0,4599)$.

Tras informarles sobre la importancia de la vacunación de acuerdo con el protocolo de actuación recomendado por el COFPO, 27 (29,4\%) de los que no pensaban vacunarse decidieron acudir al centro de atención primaria (CAP) para hacerlo, 21 $(22,8 \%)$ dudaban y $44(47,8 \%)$ no modificaron su intención de no vacunarse. No hubo diferencias significativas en relación con el nivel de estudios $(p=0,5974)$, ni con estar o no en GR $(p=0,4160)$.
Aunque no se cuantificaron al no formar parte de los objetivos del estudio, se recogieron los comentarios de los entrevistados sobre la razón de no vacunarse. Resultaron mayoritarios los referidos a la desconfianza en efectividad de la vacuna ("el único año que me vacuné cogí la gripe"), a reacciones adversas ("el año pasado me vacuné y me dio reacción"), o la falta de ejemplo de los profesionales sanitarios ("nadie me lo recomendó nunca”, "mi mujer es enfermera y no se vacuna, por lo tanto yo tampoco").

\section{Opinión de los farmacéuticos}

Se envió la encuesta a $200 \mathrm{FC}$, recibiéndose 93 respuestas (46,5\%). Las características demográficas de la muestra se reflejan en la tabla 4.

Para aumentar la tasa de vacunación 33 (35,5\%) creen que el mejor sistema sería vacunar en la farmacia, $33(35,5 \%)$ realizar campañas en la farmacia, $15(16,1 \%)$ realizar campañas y vacunar en la farmacia y 12 $(12,9 \%)$ otras acciones.

$63(67,7 \%)$ FC consideran que la farmacia comunitaria debe ser un punto de administración de la vacuna de la gripe estacional y 49 $(52,7 \%)$ creen que también debe serlo de otros tipos de inyectables. La distribución por categoría de ejercicio es de $82,4 \%$ de titulares a favor frente a 59,3\% de adjuntos ( $p=0,0221$ ), por localidad de $66,7 \%$ a favor en R, 81,8\% en SU y 57,8\% en $U(p=0,0803)$ y por pertenencia o no a SEFAC, $85,4 \%$ a favor en socios frente a 53,9\% a favor entre no socios $(\mathrm{p}=0,0012)$. Opinión positiva del $89,5 \%$ de los titulares socios de SEFAC y del $81,8 \%$ de los adjuntos socios de SEFAC.

Con respecto a la administración de otros inyectables, es favorable en el 67,7\% de titulares y en el 44,5\% de adjuntos ( $p=0,0283)$ y en el $73,2 \%$ de socios de SEFAC frente a 36,5\% en no socios $(\mathrm{p}=0,0004)$.

$82(88,2 \%)$ FC opinan que se necesita formación específica para la actividad, sin diferencias significativas entre localidad ( $p=0,753)$, categoría de ejercicio $(p=0,1871)$ ni pertenencia o no a SEFAC $(p=0,4568)$. Las entidades que consideran deben realizar dicha formación y acreditar su capacitación se muestran en la tabla 5. 
Tabla 4 Caracteristicas demográficas de los FC que respondieron a la encuesta

\begin{tabular}{|c|c|c|c|}
\hline Característica & $\begin{array}{c}\text { Hombres } \\
\text { n (\%) }\end{array}$ & $\begin{array}{c}\text { Mujeres } \\
n(\%)\end{array}$ & $\begin{array}{l}\text { Total } \\
\text { n (\%) }\end{array}$ \\
\hline Sexo & $27(29,0 \%)$ & $66(71,0 \%)$ & $93(100)$ \\
\hline Edad m (DE) & $45,8(12,2)$ & $40,6(11,2)$ & $42,1(11,6)$ \\
\hline \multicolumn{4}{|c|}{ Categoria de ejercicio profesional* } \\
\hline Titulares & $18(52,9)$ & $16(47,1)$ & $34(36,6)$ \\
\hline Adjuntos & $9(15,3)$ & $50(84,7)$ & $59(63,4)$ \\
\hline \multicolumn{4}{|c|}{ Localidad de ejercicio } \\
\hline Rural & $4(26,7)$ & $11(73,3)$ & $15(16,1)$ \\
\hline Semiurbano & $11(33,3)$ & $22(66,7)$ & $33(35,5)$ \\
\hline Urbano & $12(26,7)$ & $23(73,3)$ & $45(48,4)$ \\
\hline \multicolumn{4}{|c|}{ Pertenencia a SEFAC } \\
\hline Socios & $16(39,0)$ & $25(61,0)$ & $41(44,1 \%)$ \\
\hline No socios & $11(21,2)$ & $41(78,8)$ & $52(55,9 \%)$ \\
\hline
\end{tabular}

${ }^{*} p=0,0001$

Tabla 5 Opinión de los FC sobre las entidades que deben impartir la formación y acreditar la capacitación

\begin{tabular}{|l|c|c|}
\hline Entidad & $\begin{array}{c}\text { Formación } \\
\mathbf{n}(\%)\end{array}$ & $\begin{array}{c}\text { Acreditación } \\
\mathbf{n}(\%)\end{array}$ \\
\hline SERGAS & $22(23,7)$ & $30(32,3)$ \\
\hline COF+SERGAS & $20(21,5)$ & $19(20,4)$ \\
\hline COF+SEFAC+SERGAS & $9(9,7)$ & $7(7,5)$ \\
\hline COF+SERGAS+Universidad & $9(9,7)$ & $5(5,4)$ \\
\hline COF & $7(7,5)$ & $11(11,8)$ \\
\hline SERGAS+Universidad & $4(4,3)$ & $9(9,7)$ \\
\hline COF+Universidad & $4(4,3)$ & $4(4,3)$ \\
\hline COF+SEFAC & $4(4,3)$ & $2(2,2)$ \\
\hline Universidad & $3(3,2)$ & $1(1,1)$ \\
\hline SEFAC & $3(3,2)$ & $3(3,2)$ \\
\hline SEFAC+SERGAS & & $2(2,2)$ \\
\hline OtroS & $8(8,6)$ & $93(100)$ \\
\hline
\end{tabular}

Las opiniones de pacientes y farmacéuticos sobre quién debería realizar el pago del servicio se muestran en la figura 1.

\section{Discusión}

En el estudio que se presenta se ha querido conocer la opinión de pacientes y farmacéuticos sobre la posibilidad de administración en las farmacias comunitarias españolas de la vacuna de la gripe estacional por farmacéuticos acreditados para esa actividad. Los resultados muestran que el $85 \%$ de los encuestados acogieron favorablemente la posibilidad de recibir la vacuna en la farmacia. Los motivos por los que la prefieren a los CAP reflejan algunos de los valores que la farmacia como centro de salud aporta a la comunidad: proximidad y accesibilidad del profesional sanitario que les atiende, y coinciden con los recogidos en los estudios que valoran los beneficios de la vacunación por farmacéuticos [17-23,24,25].

Las recomendaciones de instituciones sanitarias supraestatales $[4,26]$ en el sentido de elevar las tasas de vacunación y aumentar la accesibilidad de la población a la vacuna ha llevado a muchos países de nuestro entorno a promover dicho servicio en farmacias comunitarias con personal formado con el fin de reducir el alto impacto económico, social y sanitario de la gripe estacional.

Los resultados son muy positivos, tanto en el incremento de las tasas de vacunación como en la satisfacción de los pacientes [17-23,24,25], por lo que cada vez son más los países que

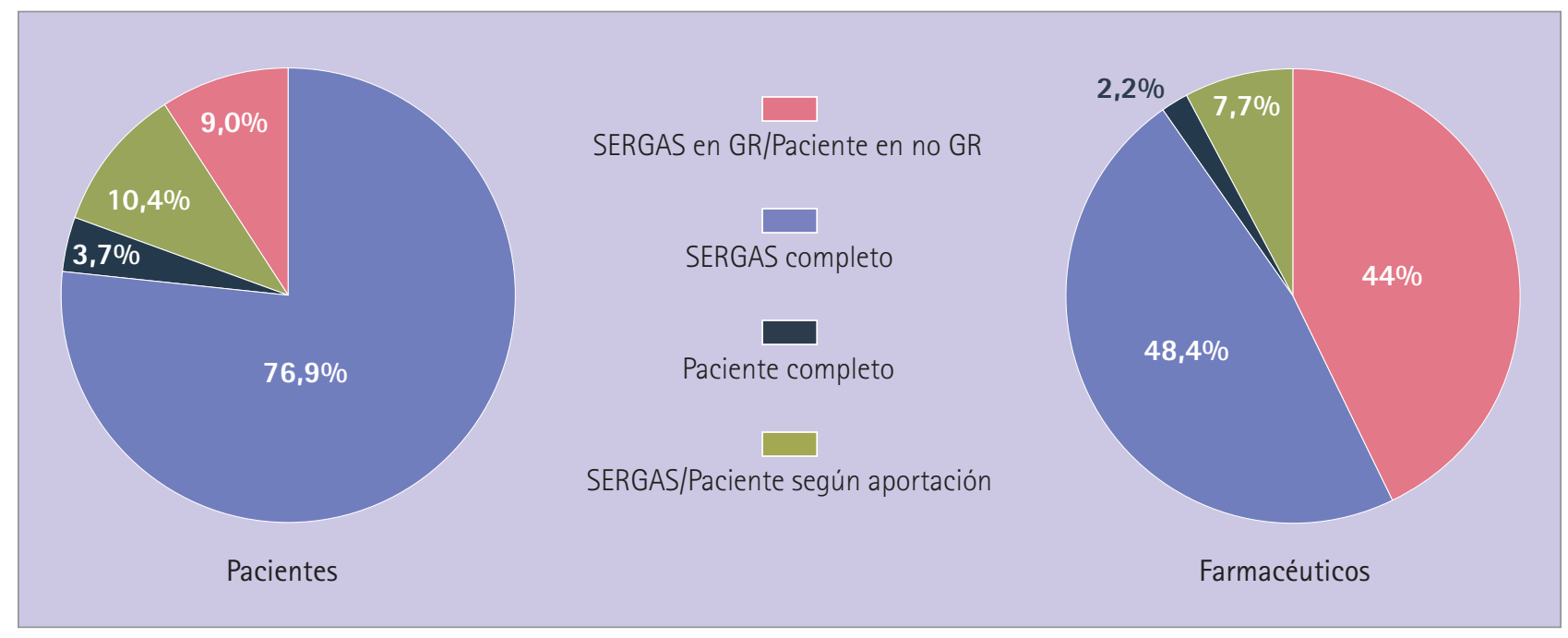

Figura 1 Opiniones de pacientes y farmacéuticos sobre quién debería realizar el pago del servicio 
incorporan la vacunación antigripal a la cartera de servicios profesionales de los farmacéuticos comunitarios que obtuvieron la capacitación adecuada o extienden sus campos de actuación [27,28].

La intervención educativa del FC, según el protocolo recomendado por el COFP0, modificó la intención de no vacunarse de casi el 30\% de los usuarios no vacunados y que no pensaban hacerlo. Si bien no se estableció en el procedimiento la comprobación de que realmente acudieran al CAP a recibir la vacuna, se trata un incremento importante de la intención de vacunarse y muestra la efectividad de la intervención educativa del farmacéutico. El porcentaje de mejora es algo superior al conseguido (24,6\%) en un trabajo que, entre otros objetivos, evaluaba también el aumento de la intención de vacunación tras intervención del FC [29]. Se trata del único estudio con características similares al nuestro, por lo que no tenemos más elementos de comparación.

Aunque hubo diferencias significativas entre los distintos niveles de estudios de los encuestados y la pertenencia o no a grupo de riesgo en cuanto a estar ya vacunados, no las hubo en relación con la intención de vacunarse antes ni después de la intervención educativa.

Las razones para no vacunarse coinciden igualmente con las encontradas en el referido trabajo [29] y con las detectadas en otros estudios con pacientes [30-32]: desconfianza en la efectividad y seguridad de la vacuna, desconocimiento de las posibles complicaciones y la no recomendación activa de los profesionales del sistema de salud. Las dos primeras son también motivos referidos por los profesionales sanitarios que explican la baja tasa de vacunación en este grupo [33-36]. Un estudio del año 2016 del Vaccine Confidence Project comprueba que Europa tiene el índice más alto de respuestas negativas en la percepción de la importancia de las vacunas, su seguridad y su eficacia, lo que conduce al nivel más elevado de reticencia a la vacunación entre la población [37]. La reducida tasa de protección que se produce algunos años [9-11], siendo la de la campaña 2017/18 una de las menores, con una protección moderada frente a gripe $B$ y subóptima ante gripe A [38], puede contribuir a esa percepción. Se nece- sita la implicación activa de todos los profesionales sanitarios que puedan contribuir a modificarla.

El porcentaje de respuestas entre los FC $(46,5 \%)$ es satisfactorio, ya que en diversos estudios mediante encuestas en línea o correo electrónico las respuestas entre profesionales sanitarios estuvieron entre el 7,3\% y el $38,8 \%[36,39,40]$, menores que la obtenida en la nuestra. La muestra coincide en sexo, localidad y categoría de ejercicio con la distribución de los FC de la provincia de Pontevedra [41].

Dos tercios de los farmacéuticos comunitarios encuestados consideran que la farmacia comunitaria es un lugar adecuado para la administración de la vacuna de la gripe estacional, aunque solo la mitad creen que debe serlo también de otros inyectables. Creen igualmente que sería el mejor sistema, junto con la realización de campañas de información en la farmacia, para aumentar la tasa de vacunación. Valoramos en conjunto como muy positiva la aceptación por los FC de Pontevedra de la posible incorporación de un nuevo servicio profesional con los matices que vamos a comentar y que no podemos comparar con otros estudios al no haber encontrado ningún otro que analice esta cuestión

No hubo diferencias significativas en cuanto a la localidad de ejercicio, pero sí entre las categorías profesionales, con un mayor porcentaje de opiniones positivas entre los titulares $(82,4 \%$ vs. 59,3\%) y entre los socios de SEFAC frente a los no socios ( $85,4 \%$ vs. 53,9\%). Podría pensarse que entre titulares y adjuntos la diferencia se debe a un diferente enfoque en el posible beneficio económico que reportaría el servicio, pero no ha sido éste el enfoque con que se ha planteado. Por otro lado, la casi unanimidad en la opinión favorable entre los socios de SEFAC incluye porcentajes similares en titulares y adjuntos. Creemos, por tanto, que la explicación de esa diferente acogida a la posibilidad de un nuevo servicio profesional está relacionada más con una falta de preparación, de motivación y expectativas profesionales y que hay un gran desconocimiento sobre qué camino debe recorrer un farmacéutico en su ejercicio profesional, lo que lleva a un cierto desaliento al comprobar que no existe un camino más allá de la propia dignidad de ejercer de manera responsable la profesión. Los socios de SEFAC encuentran esa motivación en lo que la sociedad les ofrece: formación actualizada, diseño de nuevos servicios profesionales, participación en grupos de trabajo, colaboración en proyectos de investigación, etc. Pero para muchos no es suficiente y creemos urgente la implantación de un modelo de carrera o desarrollo profesional que dé la posibilidad al farmacéutico comunitario de elevar su categoria laboral al mismo tiempo que mejora su capacitación profesional y su nivel retributivo.

La coincidencia sobre la necesidad de una formación específica para realizar la actividad es casi unánime y sin diferencias entre los encuestados. Consideran que en la formación y acreditación deben tener un papel fundamental la correspondiente administración sanitaria (SERGAS) y el Colegio de Farmacéuticos. Muy pocos creen que la Universidad debe tener un papel en dichas tareas, lo que confirma que los FC encuestados saben que esta institución no satisface las necesidades de formación para su labor diaria en la atención a la salud de las personas que acuden a las farmacias y mucho menos para la implementación de nuevos servicios profesionales de carácter asistencial que los pacientes necesitan y que constituyen el futuro de nuestra profesión.

Tanto pacientes como $\mathrm{FC}$ creen mayoritariamente que el abono del servicio de administración de la vacuna debe ser íntegro por el SERGAS, aunque un porcentaje importante de farmacéuticos opinan que en el caso de no estar incluido en GR el pago debería efectuarlo el paciente. Podría ser un sistema razonable de pago que ya existe en algunos países [22].

Una limitación del estudio es el hecho de que las encuestas fueran administradas por el FC a pacientes captados en el mostrador de la farmacia, aunque la inexistencia de rechazos a contestar y el elevado porcentaje de coincidencia en las opiniones permiten creer que este factor no resta validez a los resultados obtenidos. De igual manera podría considerarse la existencia de un sesgo de selección en el perfil de los FC que respondieron a la solicitud de participación en la encuesta, inevitable al utilizar el correo electrónico como método 
de envío/respuesta, y a pesar de la aleatorización realizada. Creemos que la coincidencia de la muestra con la distribución de los FC ejercientes en Pontevedra valida la coherencia de los resultados.

\section{Conclusiones}

La opinión de que la farmacia comunitaria debe ser punto de administración de la vacuna antigripal es ampliamente mayoritaria entre los usuarios de la farmacia.

Con la acción educativa se consiguió mejorar la intención de acudir a vacunarse de un 30\% de usuarios que no pensaban hacerlo.

La opinión de que la farmacia debe ser un punto de administración de la vacuna antigripal es también mayoritaria entre los farmacéuticos comunitarios, aunque significativamente más favorable en titulares que en adjuntos y en socios de SEFAC que en no socios.

En la formación y la acreditación para la actividad deben tener un papel fundamental el SERGAS y el Colegio Oficial de Farmacéuticos.

Tanto pacientes como farmacéuticos creen que el abono de la prestación debería realizarlo el SERGAS, aunque un porcentaje importante de FC opinan que debería pagarlo el propio paciente si no está incluido en un grupo de riesgo.

\section{Agradecimientos}

A todos los pacientes y farmacéuticos comunitarios que amablemente accedieron a participar en el estudio contestado las encuestas. Al Colegio de Farmacéuticos de Pontevedra, en la persona de su Directora del Departamento Profesional, Rosario García Riestra, que facilitó los medios para hacer llegar las encuestas a los farmacéuticos comunitarios de los municipios de la provincia mediante selección aleatoria estratificada y de manera anónima las respuestas al grupo investigador.

\section{Referencias bibliográficas}

1. Iñiguez Vázquez I, Rubial Bran D, Matesanz Fernández M, Rigueiro Veloso MT, Casariego Vales E. Gripe. [Monografía en Internet]. Guías de Práctica Clínica Fisterra. 2013. [Acceso 20/11/2017]. Disponible en: http:// www.fisterra.com/guias-clinicas/gripe/

2. Ministerio de Sanidad, Servicios Sociales e Igualdad. [Internet]. La Gripe. https://www.msssi.gob.es/ciudadanos/enfLesiones/enfTransmisibles/ gripe/gripe.htm

3. Andrés-Rodríguez NF, Fornos-Pérez JA, Mera-Gallego R. Prevención de catarros y resfriados. El Farmacéutico 2017;544:20-25.

4. World Health Organization [Internet]. Influenza (seasonal) (updated 2016, Nov). [Acceso 9/12/2017]. Disponioble en: http://www.who.int/mediacentre/factsheets/fs211/en/

5. IM Farmacias [Internet]. Combatiendo la gripe. 2016. [Acceso 8/12/2017]. Disponible en: http://www.imfarmacias.es/uploads/vacunas_medicamentos_mejores_11215_15112130.pdf

6. Red Nacional de Vigilancia Epidemiológica [Internet]. Informe de Vigilancia de la Gripe en España. Temporada 2016-2017. [Acceso 9/12/2017]. Disponible en: http://vgripe.isciii. es/documentos/20162017/InformesAnuales/Informe_Vigilancia_GRIPE_2016-2017_v.28septiembre2017. pdf

7. Coleman BL, Fadel SA, Fitzpatrick T, Thomas S-M. Risk factors for serious outcomes associated with influenza illness in high-versus low-and middle-income countries: Systematic literature review and meta-analysis. Influenza Other Respi Viruses. 2017;12(1):22-29. doi:10.1111/irv.12504

8. Centers for Disease Control and Prevention [Internet]. Key Facts About Seasonal Flu Vaccine. 2017. [Acceso 10/12/2017]. Disponible en: https:// www.cdc.gov/flu/protect/keyfacts. htm

9. Flannery B, Clippard J, Zimmerman RK, Nowalk MP, Jackson ML, Jackson LA. Early Estimates of Seasonal Influenza Vaccine Effectiveness - United States, January 2015. Morbidity and Mortality Weekly Report. 2015; 64(1):10-15.

10. Organización Mundial de la Salud [Internet]. Questions and Answers. Vaccine effectiveness estimates for seasonal influenza vaccines. February 2015. [Acceso 8/12/2017]. Disponible en: http://www.who.int/ influenza/vaccines/virus/recommendations/201502_qanda_vaccineeffectiveness.pdf

11. Servizo Galego de Saúde (SERGAS) [Internet]. Campaña de vacunacion antigripal 2017. Instrucción. [Acceso 10/12/2017]. Disponible en: https:// www.sergas.es/Saude-publica/Instrucion-campa $\%$ C3\%B 1a-vacinacion-antigripal

12. Consejo Interterritorial del Sistema Nacional de Salud [Internet]. Reco- mendaciones de vacunación frente a la gripe. Temporada 2017-2018. 28/9/2017. [Acceso 12/12/17]. Disponible en: https://www.msssi.gob. es/profesionales/saludPublica/prevPromocion/vacunaciones/docs/Recomendaciones_vacunacion_gripe. pdf

13. Santibanez TA, Zhai Y, O'Halloran A, Kahn KE, Srivastav A, Liu L, et al. Flu Vaccination Coverage, United States, 2016-17. Influenza Season. Centers for Disease Control and Prevention [Internet]. 2017. [Acceso 10/12/2017]. Disponible en: https://www.cdc.gov/ flu/fluvaxview/coverage-1617estimates.htm

14. U.S. Department of Health \& Human Services. National Vaccine Program Office [Internet]. Flu Vaccination Claims Rates by Geographic Area. 28/12/2017. [Acceso 11/1/2018]. Disponible en: https://www.hhs.gov/nvpo/ index.html

15. Organisation for Economic Co-operation and Development (OECD) [Internet]. Influenza vaccination rates (indicator). 2017. doi: 10.1787/ e452582e-en. [Acceso 11/12/2017]. Disponible en: https://data.oecd.org/ healthcare/influenza-vaccination-rates.htm

16. Campus Sanofi [Internet]. El Gripómetro. Actualizado 4/12/2017. [Acceso 10/12/2017]. Disponible en: https://www.campussanofi.es/digital/ elgripometro

17. Murphy PA, Frazee SGCJP, Cohen E, Rosan JR, Harshburgher DE. Pharmacy provision of influenza vaccinations in medically underserved communities. J Am Pharm Assoc 2012. 52:6770. doi:10.1331/JAPhA.2012.10070

18. Isenor JE, Killen JL, Billard BA, McNeil SA, MacDougall D, Halperin BA, et al. Impact of pharmacists as immunizers on influenza vaccination coverage in the community-setting in Nova Scotia, Canada: 2013-2015. J Pharm Policy Pract. 2016; 9:32. doi:10.1186/s40545-016-0084-4

19. Burson RC, Buttenheim AM, Armstrong A. Feemster KA. Community pharmacies as sites of adult vaccination: A systematic review. Hum Vacc Immunother. 2016; 12(12):31463159. doi:10.1080/21645515.2016.12 15393

20. Jacinto IP, Costa S, Horta MR, Mendes Z, Torre C, Guerreiro JP, et al. Serviço de vacinação nas farmácias portuguesas. Rev Port Farmacoter. 2015; 7:160-166.

21. Kirkdale CL, Nebout G, Megerlin F, Thornley T. Benefits of pharmacist-led flu vaccination services in community pharmacy. Ann Pharm Fr. 2017; 75(1):3-8. doi:10.1016/j.pharma.2016.08.005 
22. Kirkdale CL, Neboutb G, Taitel M, Rubin J, Jacinto I, Horta R, et al. Implementation of flu vaccination in community pharmacies: Understanding the barriers and enablers. Ann Pharm Fra. 2017; 75(1): 9-16. doi:10.1016/j. pharma.2016.08.006

23. Astier A. La vaccination antigrippale à l'officine. Ann Pharm Fra. 2017; 75(1):1-2. doi:10.1016/j.pharma.2016.12.002

24. Hook S, Windle J. Community pharmacy influenza immunisation increases vaccine uptake and gains public approval. Aust $\mathrm{N} \mathrm{Z} \mathrm{J} \mathrm{Pu}-$ blic Health. 2013; 37(5):489-490 doi:10.1111/1753-6405.12109

25. Drozd EM, Miller L, Johnsrud M. Impact of Pharmacist Immunization Authority on Seasonal Influenza Immunization Rates Across States. Clin Therapeutics. 2017; 39(8): 1563-1580. doi:10.1016/j.clinthera.2017.07.004

26. Organisation pour le Developement et la Cooperation Europeénne (OECD) [Internet]. Panorama de la santé 2017: Les indicateurs de l'OCDE. Paris: Éditions OCDE, 2017. [Acceso 11/12/2017]. Disponible en: http:// dx.doi.org/10.1787/health_glance2017-fr

27. Correo Farmacéutico [Internet]. Todas las farmacias francesas podrán vacunar de la gripe en 2019. Sección Farmacia Internacional 26/3/2018 [Acceso 28/6/2018]. Disponible en: https://www.correofarmaceutico.com/farmacia-internacional/ todas-las-farmacias-francesas-podran-vacunar-de-la-gripe-en-2019. html

28. Correo Farmacéutico [Internet]. Farmacéuticos de Nueva York podrán vacunar a niños frente a la gripe. Sección Farmacia Internacional 29/1/2018. [Acceso 28/6/2018]. Disponible en: https://www.correofarmaceutico. com/farmacia-internacional/farmaceuticos-nueva-york-vacunar-ninos-gripe.html

29. Aparicio C, Albanell F, Bellver S, Colmenero L, del Cacho MS, López P, et al. Efectividad de la recomendación del farmacéutico comunitario sobre la vacunación antigripal en pacientes incluidos en grupos de riesgo. Farmacéuticos Comunitarios. 2018; 10(Supl.1):103

30. Sarriá A, Timoner J. Determinantes de la vacunación de la gripe en personas mayores de 65 años. Rev Esp Salud Pública. 2002; 76(1):17-26.

31. Sagor KH, AlAteeq, MA. Beliefs, attitudes' and barriers associated with the uptake of the seasonal influenza vaccine among patients visiting primary healthcare clinics. Saudi Med J. 2018; 39(7):690-696.

32. Casalino E, Ghazali A, Bouzid D, Antoniol S, Pereira L, Kenway P, et al. Patient's behaviors and missed opportunities for vaccination against seasonal epidemic influenza and evaluation of their impact on patient's influenza vaccine uptake. PLoS One. 2018 Mar 22;13(3):e0193029. https://doi.org/10.1371/journal. pone.0193029

33. Sánchez-Payá J, Hernández-García I, Barrenengoa Sañudo J, Martínez HR, Camargo Angeles R, Cartagena Llopis $\mathrm{L}$, et al. Determinantes de la vacunación antigripal en personal sanitario, temporada 2009-2010. Gac Sanit. 2011; 25(1):29-34. doi:10.1016/j.gaceta.2010.09.018

34. Muncunill M, Bosch MA, Tamborero G, Fonseca M. Factores condicionantes de la vacunación antigripal en profesionales de atención primaria. Gac Sanit. 2011;25(5):439-440. doi:10.1016/j.gaceta.2011.04.014

35. Domínguez A, Godoy P, Castilla J, Mayoral JM, Soldevila N, Torner N. Knowledge of and attitudes to influenza in unvaccinated primary care physicians and nurses. A cross-sectional study. Hum Vaccin Immunother. 2014; 10(8):2378$2386 . \quad$ https://doi.org/10.4161/ hv.29142

36. Toledo D, Soldevila N, Guayta-Escolies R, Lozano P, Rius P, Gascón P, et al. Knowledge of and Attitudes to In- fluenza Vaccination among Community Pharmacists in Catalonia (Spain). 2013-2014 Season: A Cross Sectional Study. Int. J. Environ. Res. Public Health 2017; 14:756. doi:10.3390/ ijerph14070756

37. Larson HJ, de Figueiredo A, Xiahong Z, Schulz WS, Verger P, Johnston IG. et al. The State of Vaccine Confidence 2016: Global Insights Through a 67-Country Survey, EBioMedicine. 2016; 12:295-301. doi:10.1016/j. ebiom.2016.08.042

38. Centro Nacional de Epidemiología y Centro Nacional de Microbiología [Internet]. Efectividad de la vacuna antigripal 2017-2018 en España. Estimaciones preliminares, semana 44/2017-semana 03/2018 (30 de octubre - 21 enero 2018). Febrero 2018. [Acceso 12/6/2018]. Disponible en: http://www.isciii.es/ISCIII/ es/contenidos/fd-servicios-cientifico-tecnicos/fd-vigilancias-alertas/ fd-enfermedades/pdf_2018/CNE_ISCIII_Efectividad_vacuna_antripal_2017_18_Estimaciones_Preliminares_9_Febrero2018.pdf

39. Aerny N, Domínguez MF, Astray J, Esteban-Vasallo MD, Blanco LM, López MA. Tasas de respuesta a tres estudios de opinión realizados mediante cuestionarios en línea en el ámbito sanitario. Gac Sanit. 2012; 26(5):477-9. doi:10.1016/j.gaceta.2011.10.016

40. Torner N, Godoy P, Soldevila N, Toledo D, Rius C, Domínguez A. Estudio actitudes sobre vacunación antigripal en profesionales sanitarios de atención primaria de Cataluña. Aten Primaria. 2016; 48(3):192-199. https:// doi.org/10.1016/j.aprim.2014.09.014

41. Consejo General de Colegios Oficiales de Farmacéuticos [Internet]. Estadísticas de Colegiados y Farmacias Comunitarias 2017. [Acceso 24/6/2018]. Disponible en: http:// www.portalfarma.com/Profesionales/ infoestadistica/Documents/Documentos-acceso-medios/Estadisticas-Colegiados-Farmacias-Comunitarias-2017.pdf 
Anexo 1. a) Encuesta a pacientes

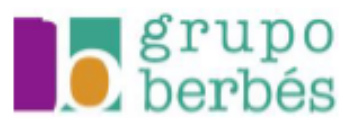

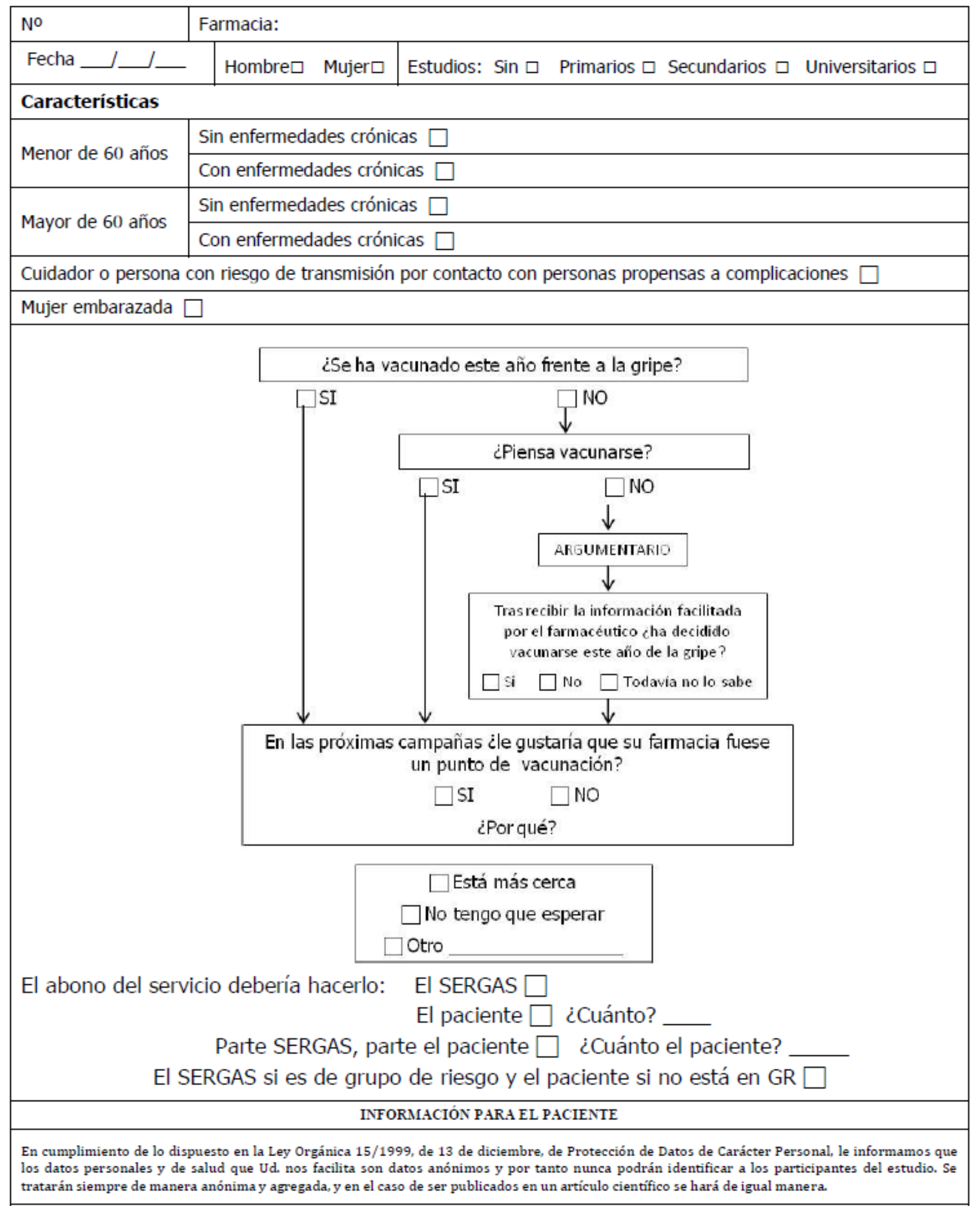


Anexo 1. b) Encuesta a farmacéuticos

D. Girupo

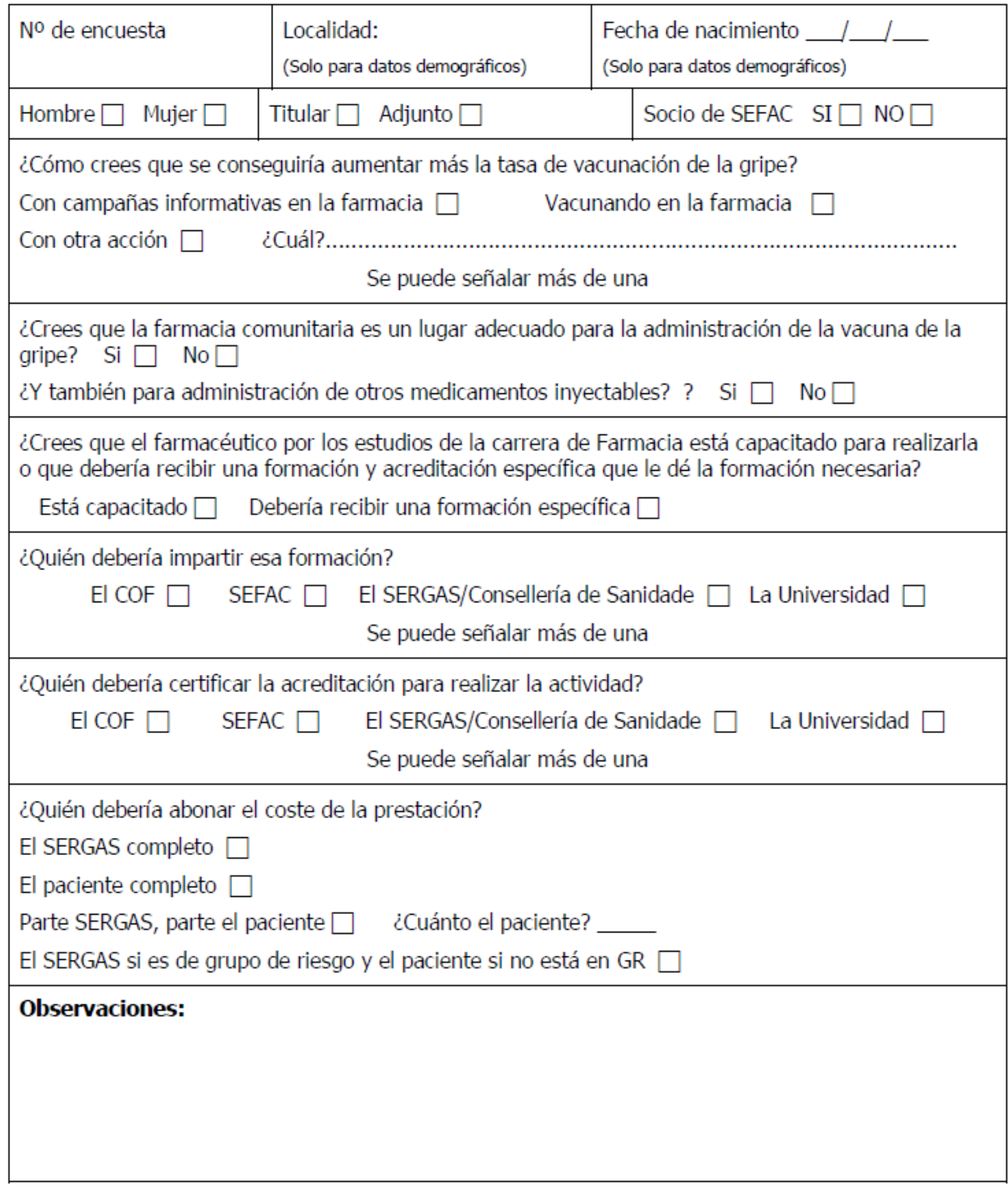

La encuesta se tratará de manera anónima y agregada, sin identificar al farmacéutico que la cumplimenta. 